documentation. Or, il était aussi facile de faire bien que mal, puisque, dans les deux cas, il n'y avait qu'à copier (I).

Je ne parle pas de ceux qui, une fois citéc "l'Ephémère de Mai ", ont à peu près épuisé leur bagage éphéméroptérologique scientifique, mais, en revanche, nous accablent de la richesse - anglaise - de leur terminologie halieutique. La possession, mème totale, de ce vocabulaire ne servirait à rien à l'aménagiste. J'en connais qui tentèrent vainement de rechercher à quoi pouvaient bien correspondre scientifiquement les " imitations " tant vantées.

J'ai eu, jadis, l'occasion de recevoir de fort belles planches sur lesquelles je devais reconnaître les Ephéméroptères coloriés. Tout au plus, en tant que spécialiste, ai-je pu déterminer les genres. Je crois que ni l'auteur, ni surtout l'artiste, n'avait la moindre notion de ce qui eût pu faire différencier les espèces. N'ayant jamais vu le texte, j’ignore la part du vrai et du faux de cet ouvrage, et si l'aménagiste y trouverait autant d'avantages que le simple pêcheur beaucoup moins difficile.

(A suivre).

\title{
L'ART D'ÉLEVER LES POISSONS D'AQUARIUM
}

\author{
Par M. Marcel DAGRY
}

(Suite) ${ }^{(2)}$

L'aquarium étant bien préparé, il reste à le peupler.

On distingue, parmi les poissons d'ornement qui se diputent la faveur des amateurs, deux grands groupes dont il sera traité séparément : - les vivipares et les ovipares.

\section{I. - Poissons exoliques vivipares.}

Les espèces vivipares ou ovovivipares sont celles pour lesquelles la femelle accouche de petits vivants, bien constitués, qui se mettent à nager et même à manger aussitôt après leur naissance.

C'est une erreur de croire ces espèces exclusivement marines; bon

(1) C'est, entre autres, le cas du Dictionnaire de LA Buaviònt (supplément de ig26), oì se trouve la plus belle salade éphéméroptérienne qui soit, par ignorance de ce qui a été publié depuis Picter.

(2) Voir Bulletin; - $\mathbf{n}^{\circ} 7^{2}$, Juin 1934 , p. $328 ;-\mathrm{n}^{\circ} 73$, Juillet, p. $\mathbf{8} 8$. 
nombre d'entre elles sont fluviatiles et se rencontrent dans les eaux chaudes des contrées équatoriales, en $\Lambda$ sie, en Afrique et en Amérique.

Rien de plus intéressant à observer que la reproduction de ces poissons et de voir, en particulier, leur progéniture, aussitôt sortie du corps de la mère, se répandre dans tout l'aquarium, se réfugiant parmi les herbes aux endroits les plus touffus. Ces petits savent, semble-t-il, que les auteurs de leurs jours, quelque peu cannibales, mettraient un terme rapide à leur précaire existence s'ils ne s'en écartaient rapidement.

Les vivipares ont tous, plus ou moins, ce terrible défaut d'être friands de leurs enfants ; aussi emploie-t-on, pour y remédier, divers procédés.

La première des choses est de faire, dans les bacs où l'on entreprend un élevage, une plantation serrée de Myriophyllum, qui occupera au moins la moitié de la contenance. On installera, ensuite, des accouchoirs où sont

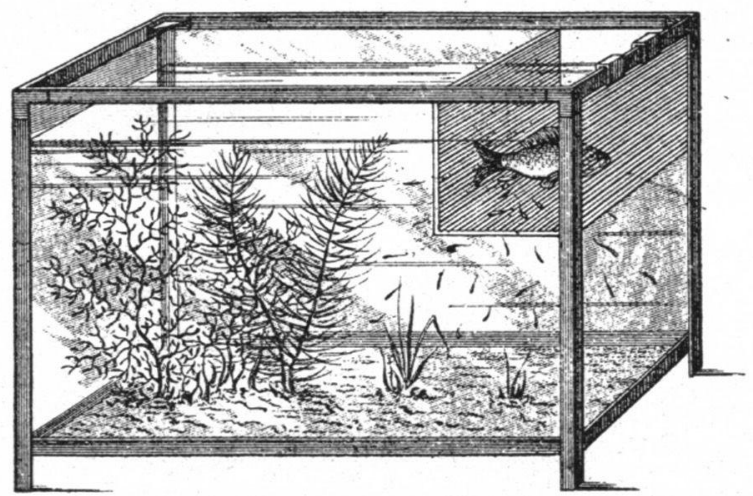

Fig. 13. - Aquarium de vivipares avec accouchoir.

enfermées les mères au moment où elles arrivent à terme (fig. 13). Les alevins seuls peuvent passer entre les baguetles de cette sorte de cage pour aller trouver abri et pâture dans les herbes où ils prennent rapidement force et vigueur. Ils sont, d'ailleurs, des plus faciles à élever, puisque déjà complètement formés à la naissance ; leur appétit se manifeste presque immédiatement. A une température de $20^{\circ}-25^{\circ} \mathrm{C}$., - qui est, selon les espèces, celle nécessaire pour la reproduction - et bien nourris, ils deviennent adultes en moins de six mois.

L'organe d'accouplement, chez le mâle, se présente sous la forme d'un appendice tubuleux, conique et effilé, placé sous le ventre légèrement à l'avant des ventrales, et allongé d'avant en arrière. Lorsqu'il devient fonctionnel, il tourne autour de son insertion qui forme charnière et pointe alors vers l'avant. Le poisson fonce ainsi sur la femelle qui, pendant lia saison des amours seulement, se prête au rapprochement et le facilite en incurvant légèrement son corps et en nageant sur le côté. La fécondation s'effectue par simple mise en contact, sans pénétration, des orifices génitaux. Les attaques se répètent autant qu'il est nécessaire et, au bout de 
quelques jours, on voit le ventre de la femelle grossir ct une teinte d'un bleu foncé ardoisć en colorer la peau distenduc. C'est là le signe caractéristique de la grossesse ; le manège amourcux cesse et les époux semblent se fuir.

Quand le moment de la délivrance approche, la femelle parcourt l'aquarium en tout sens, avec une sorte d'affolement, en suivant les parois d'une nage rapide ascendante et descendanle. C'est le moment où la surveillance doit s'exercer. Il est prudent d'enlever le père et d'isoler la future mère dans un compartiment du bac, au moyen d'une sorte de cage, si l'on veut conserver la presque totalité de sa descendances.

Dans le cas contrair, il est curieux de voir le jeu du mâle.

A nouveau il se rapproche de la femelle et, par des pressions buccales sur son abdomen, facilite la délivrance. Mais ce n'est pas par amour palerncl ou conjugal qu'il intervient : il agit sous l'impulsion de la faim ; car les parents sont, comme il a été signalé plus haut, fort enclins à dévorer leurs enfants au fur et à mesurẹ de leurs naissances. Heureusement !a Nature, suprême sauvegrarde des races et espèces, a donné à ces petits ĉtres linstinct de conservation et on les voit, dès leur sortic du ventre maternel, nager vigoureusement pour gagner un abri sûr parmi les plantes où, sans faire de mouvements pouvanl trahir leur présence, ils trouvent asscz de nourriture pour passer les 3 ou 4 premiers jours de leur existence. $\Lambda u$ bout de ce temps, ils sont à l'abri du danger et sortent de leur retraite. S'ils n'ont plus à craindre leurs parents, ils n'ont rien, non plus, à en attendre. Ce n'est pas comme chez certains ovipares où le mâle veille assidûment sur sa progéniture, la défend, l'alimenle, après avoir, préalablement, préparé le nid qu'elle doit quitter seulement avec sa permission et sous sa conduite.

Malgré le déchet qu'implique l'indifférence on l'hostilité des parents, malgré les difficultés de la lutte pour l'existence, les espèces vivipares sc maintiennent grâce à leur prolificilé. Une femelle adulte donne naissance, même en aquarium, à des petits dont le nombre varie de ro à 200 selon lcur grosseur ; les portées se succèdent, durant la saison chaude, tous les quarante jours environ; il y en a donc 5 à 6 par an.

L'instinct destructeur des père et mère ne s'obscrve pas chez toutes les espèces vivipares ; mais surtout parmi les variétés américaincs ; celles d'Asie et d'Afrique sont moins cannibales ou même pas du tout. Ainsi, chez l'Hemiramphus fluviatilis, les géniteurs ne s'altaquent pas à leurs petits, qui nagent à leurs côtés, les suivent dans leurs ébats, sans cxciter leur voracité, mais aussi sans reccvoir d'eux le moindre soin.

Les petits naissent entièrement développés, munis de toutes leurs nageoires ; le sexe n'est toulefois marqué qu'au bout de I à 2 mois pour certains, beaucoup plus tard pour d'autres. On peut, dès la naissance, donner comme nourriture des Daphnies et, pour varier les repas, du cour de bocuf cru râpé, des vers de vase hachés, de la pulpe de rate. 
Par ailleurs, on entretiendra dans l'aquarium d'ćlevage un assez grand nombre de plantes aquatiques, dont les feuilles tendres et les jeunes pousses sont broutées. Cette alimentation végétale est absolument nécessaire à la croissance rapide et assure aux poissons des couleurs resplendissantes et une vitalité superbe.

Tout ce qui précède vaut pour toutes les espèces vivipares. Nous allons, maintenant, décrire cerlaines des plus communes el cles plus réputécs.

Xiphophorus Helleri (IIeckrl) (Fig. 14).

Originaire du Mexique, avec des variétés spéciales au Guatémala, au Honduras et au Brésil, le Xiphophore, de la famille des Paciliides, est un des plus gracieux holes de l'aquarium d'appartement et celui à recommander aux amateurs débutants.

Comme la plupart des vivipares, il est polygame et un seul mâle féconde facilement, surtout si la température de l'eau est maintenue à $22^{\circ}-24^{\circ} \mathrm{C}$., unc douzaine de femelles ; ces dernières, si elles sont de bonne taille, mettent au monde jusqu'à ío pelits chacune dans lespace d'une heure.

Ce charmant animal joint à celte fécondité remarquable une rusticité à toute épreuve; il se familiarise très facilement et reconnait ccux qui prennent soin de lui.

Je corps est de contour général ovale ; la courbure du dos étant peu accentuée, la bouche, qui s'ouvre vers le haut, permet au poisson de saisir les moustiques sans bouger de la position horizontale. Cette disposition se remarque chez la plupart des vivipares et leur a valu l'appellation très justifiée de "gobe-mouches".

Le mâle, dont la longueur est comprise entre 8 et i 2 centimètres, se distingue à première vue par la forme tout à fait spéciale de la caudale dont le lobe inférieur, au lieu d'être, comme d'ordinaire, semblable au supéricur, s'étire en longue pointe ayant l'apparence d'une épée flexible ; cette particularité a valu à l'animal son nom qui, tiré du grec, signifie porteglaive.

Le mâle est seul aussi à avoir une livrée brillante. Ia teinte brun-olive du dos s'atténue et passe au vert sur les flancs, puis au blanc laiteux sur l'abdomen. Une bande rouge vif marque la ligne latérale, une autre la partie médiane de l'épée ; elles se relient par un raccord bleu foncé. Il existe, d'ailleurs, beaucoup de varićtés de cette espèce, dont la tonalité dominante est tantôt rouge, tantôt jaune, tantôt intermédiaire. Il s'agit, dans ce dernier ras, de croisements ou dégénérescence des types fondamentaux.

La femelle a une taille de 6 à ı contimètres ; son corps est plus épais ; l'abdomen renflé, surtout durant la giestation, la caudale symétrique. Ciependant quẹlques spécimens âcés portent un embryon d'épée, ce qui ne semble pas constituer une anomalie, étant fréquent, dans la nature. que les femelles, en vieillissant, prennent certains caractères masculins. 
La livréc féminine est analogue à celle du màle, mais avec coloris moins vif ; les nageoires sont jaunâtres et la dorsale maculéc de taches brunes, alignées, à contours dégradés.

Le Xiphophore exige une température de $18^{\circ} \mathrm{C}$. au moins ; il se reproduit lorsqu'elle atteint $22^{\circ}-24^{\circ} \mathrm{C}$. ; les portées sont de 100 à I jo petits qui naissent en 2 à 3 heures et fuient immédialement père et mère pour ṇe pas leur servir de proies. Mais ce cannibalisme cesse au bout de 2 à 3 jours.

Ce poisson est peu difficile et acceple toutes sortes de nourritures :

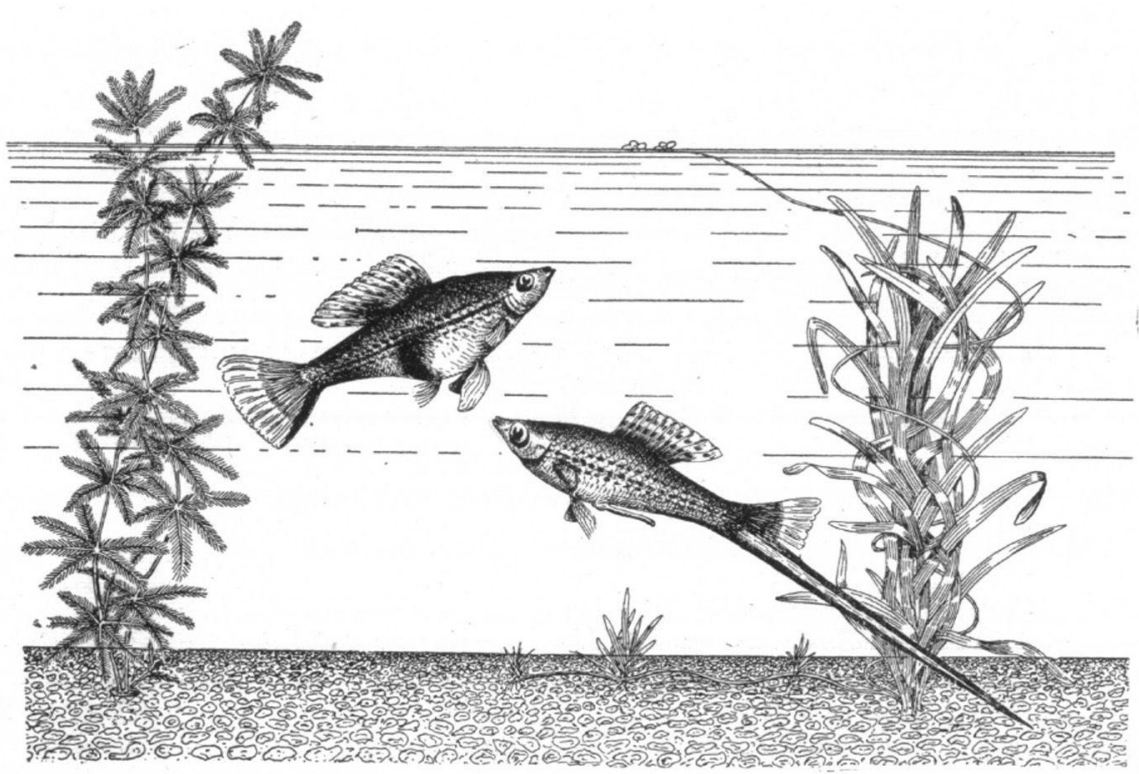

FIG. 14. - Xiphophorus Helleri (Heckel).

A droite, le mále; ì gauche, la femelle.

vers de vase ou vers de terre finement hachés, pulpe de rale de bouf on cheval, etc... ; omnivore, il se développe aussi aux dépens des plantes aquatiques.

Vivant en bonne intelligence avec les autres vivipares, le Xiphophore se recommande encore par là à l'attention du pisciculteur en chambre : jl faut noter, toutefois, que le mâle ne s'entend pas avec les autres de la même espèce.

On peut obtenir des métis, dont le coloris surpasse en beauté celui des parents, en croisant un mâle Xipho avec une belle femelle de Platy, poecilia. autre genre de la même famille ; nous parlerons plus loin de rette belle variété.

(A suivre). 